\title{
THE ELECTRO-ENCEPHALOGRAM IN CONVULSIONS INDUCED BY CARDIAZOL
}

\author{
BY \\ L. C، COOK AND W. GREY WALTER \\ From the Bexley Hospital for Nervous and Mental Disorders, and Central Pathological \\ Laboratory of the London County Hospitals for Nervous and Mental Disorders
}

\section{(ReCeived 9TH MaRch 1938)}

SEIZURES induced by cardiazol (pentamethylene tetrazol) are not identical with Grand Mal, but the clinical resemblance is sufficiently close to justify their study by electro-encephalography. Gibbs, Gibbs, and Lennox (1937) have shown conclusively that a genuine epileptic fit is accompanied by a rhythmic, high-potential electrical discharge from the cortex, and Golla, Graham, and Walter (1937) have produced evidence that the majority of young idiopathic epileptics exhibit a focal resting discharge which, by analogy with the slow waves found in the neighbourhood of cerebral tumours (Walter, 1937) have been called "delta foci." Moreover, when an epileptic patient with such a focus has a fit during electro-encephalography, the large waves characteristic of the fit appear first in or near the delta focus and spread thence to the rest of the cortex. Now in the majority of epileptics who show such a focus and complain of frequent major fits, the focus is in the region of the superior frontal gyrus, sometimes on one side only, sometimes on both. This area corresponds roughly with the "Area $6 a \beta$ " of Vogt, to which Foerster (1936) and others have attributed an "epileptogenic" function. The anatomy and physiology of this part of the cortex and its relation to other parts are not yet sufficiently clear to warrant the adoption of any definite hypothesis, but there is evidence from several sources that this part of the frontal lobe is concerned in some way with the development of convulsive seizures. The possibility of taking records during a series of controlled convulsions induced in the course of treatment of schizophrenic patients at Bexley Hospital seemed an excellent opportunity for collecting information on this matter.

The equipment was the same as that used by one of us for the study of cerebral tumours (Walter, 1937). It consists of a three-channel amplifier and triple cathode-ray oscillograph unit; the amplifiers have balanced input stages and are therefore entirely independent of one another, and the oscillographs have screens with a long after-glow which makes the observation of slow changes easy, if it is not desired to take records. With this apparatus the place of origin of a cortical discharge can be located to within a centimetre or 
so by observing the phase relations of the potential changes in the three leads. A more detailed explanation of this technique is given in the paper referred to above.

The first difficulty encountered was that some of the patients, by reason of their condition, were unwilling to co-operate and violently opposed the fitting of the cap and electrodes. This meant that many records were spoiled by the movements of the patients or the restraining efforts of the assistants. The second obstacle was that during the intravenous injection of the cardiazol the patient's arm must be firmly held, and at the beginning of the convulsion a gag must be placed in the mouth, so that even if there is no struggling, the patient must be touched twice at least, which, as is well known to those who work with amplifiers, is liable to introduce large artefacts into the record, particularly since the patient is not at earth potential when balanced input amplifiers are used. The size of these artefacts was reduced by the nurses wearing rubber gloves when touching the patient.

Sixteen patients were under treatment during the course of the experiments, and of these 14 were found sufficiently co-operative. Observations were made during 32 injections and satisfactory records were obtained from 12 typical convulsions. Artefacts from the sources mentioned above spoiled 13 of the experiments and in five cases the patient failed to have a fit.

The results obtained were the same in all the experiments in which the effect of the injection was comparable. They may be divided into two groups : first, those cases in which the injection failed to induce a convulsion, and secondly, those in which the injection was followed by a typical general convulsion. In all cases records were taken before the injection, first with high amplification, in order to observe the character of the resting electro-encephalogram, and then with the much lower amplification, which was used later to show the very large potentials which were expected to accompany the fit. When the needle was in the vein, continuous recording or observation was started and continued until either the convulsion was over or it was clear that the patient was not going to have one.

The patient whose electro-encephalogram is here illustrated was a married woman of 26, suffering from a schizophrenic illness of 2 years' duration. On admission she was depressed and apprehensive, introverted, and so preoccupied with morbid ideas as to be practically inaccessible to the usual inquiries. Hallucinations and schizoid grimacings and mannerisms were always in evidence, whilst later typically hebephrenic symptoms were superadded. Her physical condition was good and there was no evidence of neurological lesion.

On 26th June, 1937, a course of cardiazol was started, with the result that after five fits she was in a state of very fair remission and was perfectly cooperative as regards electro-encephalography.

\section{Description of Fits induced by Cardiazol}

The fits are produced by rapid, intravenous injection of a 10 per cent. ardiazol solution into one of the antecubital veins. They are in the nature of 
typical, strong, generalized epileptic convulsions. The first sign, which is usually a short cough, blepharospasm, or mouth twitching, occurs from 3 to 15 seconds after the end of the injection. Large, myoclonic jerkings of head, limbs, and body follow ( 8 to 20 seconds after the injection) and usually last from 2 to 10 seconds. The tonic phase usually begins 10 to 30 seconds after the injection and is always very severe. Its duration varies from 5 to 20 seconds, when it merges into the clonic phase, which starts approximately 18 to 40 seconds after the injection and generally lasts from 25 to 55 seconds. Flaccidity, sometimes interrupted by an occasional generalized myoclonic jerking, usually appears from 55 to 90 seconds after the injection. The pupils are nearly always dilated throughout the fit and the eyes often show extreme lateral deviation, to either side even in the same case. Other frequent accompanying features are goose-skin, micturition, and spontaneous extensor plantar sign.

In the case illustrated nine fits were induced between 26th June and 23rd July, 1937. The first three fits followed a dose of $0.5 \mathrm{gm}$. cardiazol (5 c.c. of the 10 per cent. solution), the fourth fit required $0.6 \mathrm{gm}$., the fifth fit $0.7 \mathrm{gm}$., and the last four fits, $0 \cdot 8 \mathrm{gm}$. On 12th and 13th July, 1937, electro-encephalograms were taken. On 12 th July, $0.7 \mathrm{gm}$. cardiazol was administered without producing a major fit. Except for a blank, staring expression and widening pupils, no signs appeared until 20 seconds after the end of the injection, when prolonged blepharospasm occurred. There were no convulsive movements, but for about 2 minutes short, quick, blowing respirations were in evidence. During this period the patient was semi-conscious and mentally inaccessible.

On the following day the injection of $0.8 \mathrm{gm}$. cardiazol produced a typical fit, ushered in by a short cough 5 seconds after the end of the injection, and followed immediately by blepharospasm ( 7 seconds). The tonic phase occurred at 17 seconds, clonus at 35 seconds, and the patient became flaccid at 50 seconds. There was deviation of the eyes upwards and to the right.

The experiments in which no fit followed the injection are of some interest. A typical observation was as follows. For a few seconds following the injection no change is seen in the electro-encephalogram, then small waves appear in all leads (Fig. 1). These have a frequency of about 6 per second and involve all areas of the cortex. They increase gradually in size for about a minute, and remain for some hours. During the first few minutes the patient is confused and may exhibit behaviour of the " epileptic equivalent" type, whilst later there may be feelings of uneasiness and distress lasting several hours and the symptoms of the psychosis may be more pronounced than before the injection. The electro-encephalogram does not resemble in any way that found in cases of Petit Mal, for this condition is characterized by a focal discharge at a frequency of about 3 per second, while the sub-convulsive doses of cardiazol produce a general discharge at about twice that frequency. A more detailed description of these cases is contained in a paper by one of us (Cook, 1938) on the clinical aspects of the treatment.

When the injection induces a convulsion, the electro-encephalogram starts in the same way after a latency of a few seconds (Fig. 2). The waves appear in all areas, but grow more rapidly and have a slightly lower frequency ; at about 
15 seconds after the injection there is an abrupt increase in size and drop in frequency of the waves in the frontal region. Sometimes this occurs first on the left, sometimes on the right, sometimes it appears almost simultaneously on both sides. At this moment the tonic stage of the convulsion starts. The area involved by large slow waves increases and spreads over the greater part of the cortex, then, as the fit passes into the clonic stage, the discharge becomes more staccato in rhythm with the jerks, then suddenly ceases from 1 to $1 \frac{1}{2}$ minutes after the injection. The electro-encephalogram is now abnormally quiet, no waves of any sort being seen, and the patient is completely relaxed and flaccid. As consciousness returns the normal small random potentials reappear, and 30 minutes later neither patient nor electro-encephalogram shows any trace of the storm which has agitated them.

The striking feature of the records in these convulsions is that, although the diffuse discharge starts in the same way as when no fit follows, there is a sudden increase in potential and drop in frequency in one area. Records taken with electrodes suitably placed for localizing in the frontal lobes show that the area where this sudden outburst occurs is in the neighbourhood of the superior frontal gyrus on one side or the other. The rapidity of development of the seizure prevented any correlation being established between the side of the electrical change and the side on which the convulsion started. Clinically the fits do not suggest any focal origin in the usual sense, any more than does a typical major attack, but in every case in which a satisfactory record or observation was made, there was a definite focal discharge as a prelude to the convulsion, and the focus in every case was in approximately the same relative position as the resting delta foci of idiopathic epilepsy.

In order to account for this observation, it cannot be supposed that cardiazol has an entirely selective action on one part of the cortex, since small doses produce an abnormal discharge in all areas : it may be that when a certain concentration of cardiazol is reached, this frontal area becomes unstable and acts as a detonator for the rest of the cortex, which is itself already poisoned by the drug. It should be remembered that the electrical threshold for convulsive movements has been found to be lower in this area than in other parts of the brain. Another possibility is that this area, by reason of its internal anatomical connections, is the first to signal the onset of an abnormal condition in some sub-cortical centre. In any case, in view of the prevailing uncertainty as to the normal function and connections of this part of the central nervous system, the precise relation between the electro-encephalographic changes and the motor and psychic disturbances must remain, for the time being, a subject for speculation.

\section{Summary}

Electro-encephalograms have been taken from patients undergoing treatment by cardiazol injection for schizophrenia.

When an injection failed to induce a fit an abnormal discharge was found in 
all areas of the cortex. This lasted for some hours and corresponded with a period of mental confusion and malaise.

When the injection was followed by a convulsion, the diffuse discharge was interrupted by a focal outburst coincident with the onset of the tonic spasm. This focus was always in the region of the superior frontal gyrus. When the fit was over the electro-encephalogram soon resumed its normal character.

Our sincere gratitude is due to Professor F. L. Golla, director of the Central Pathological Laboratory, and to the medical superintendent and staff of Bexley Hospital for their assistance in making these observations.

\section{REFERENCES}

Bumke, O., and Foerster, O. (1936). Handbuch der Neurologie, Springer, Berlin.

Cook, L. C. (1938). Proc. roy. Soc. Med. (in Press).

Gibbs, F. A., Gibbs, E. L., and Lennox, W. G. (1937). Brain, 60, 377.

Golla, F. L., Graham, S., and Walter, W. G. (1937). J. ment. Sci., 83, 137.

Walter, W. G. (1937). Proc. roy. Soc. Med., 30, 579. 

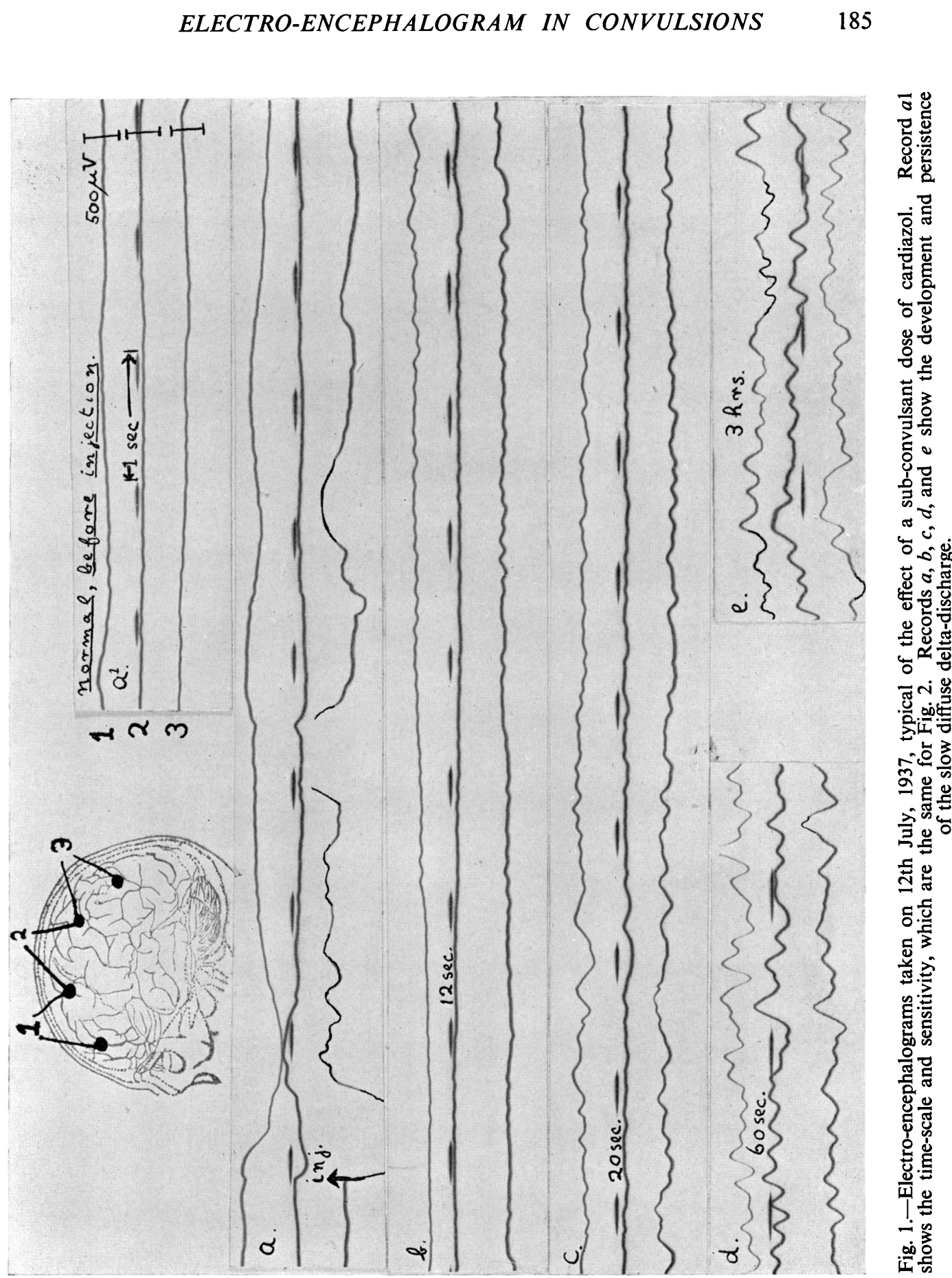


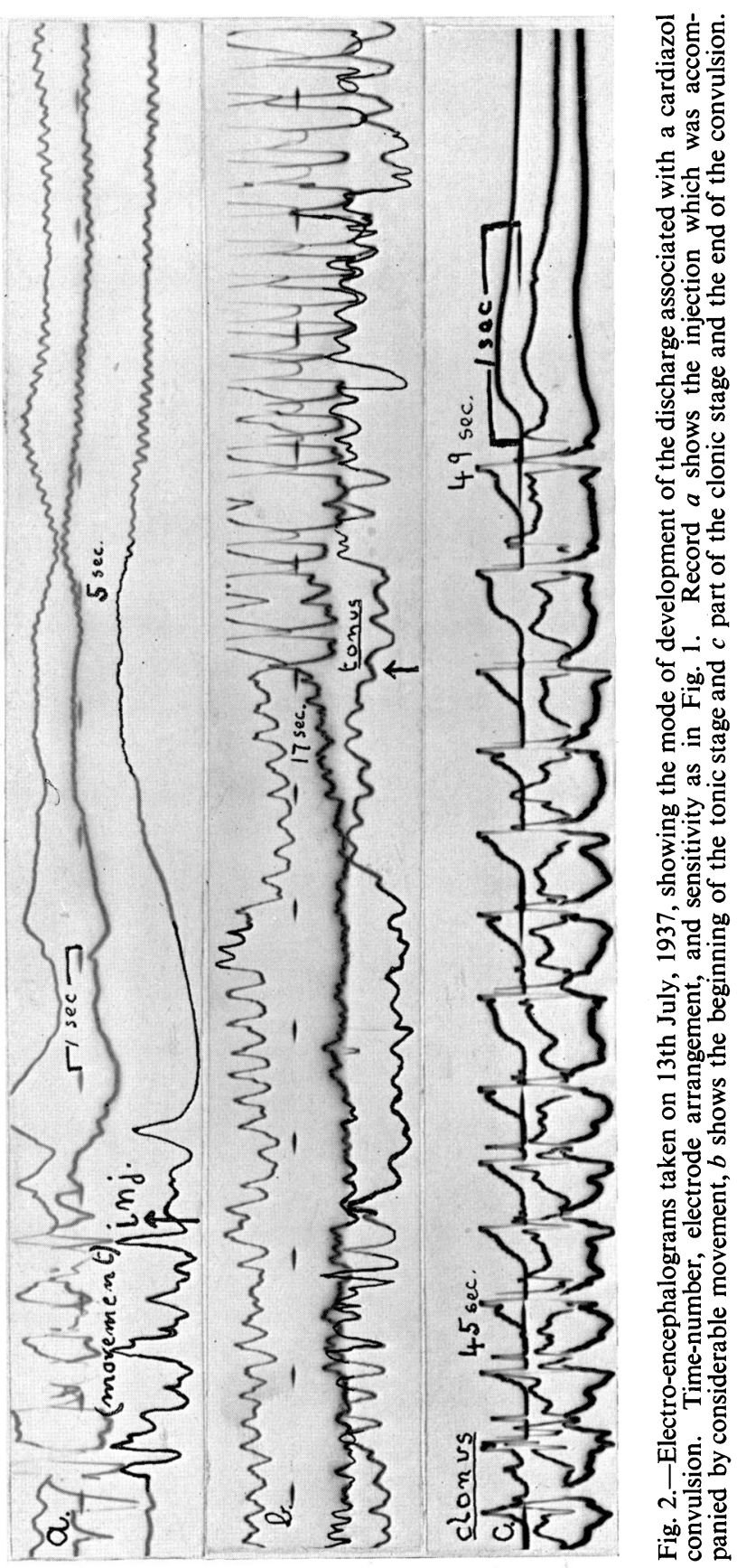

